J.Lake Sci. (湖泊科学), 2016, 28(1): 170-177

DOI 10. 18307/2016. 0120

(C) 2016 by Journal of Lake Sciences

\title{
热带浅水湖泊后生浮游动物群落结构对生态修复的响应”
}

\author{
曾海逸 ${ }^{1}$, 钟 萍 ${ }^{1}$, 赵雪枫 ${ }^{2}$, 李 超 $^{2}$, 何小林 ${ }^{2}$, 刘正文 ${ }^{1,3 * *}$ \\ (1: 暨南大学生态学系与水生生物研究中心, 广州 510632) \\ (2: 广州贝山环保科技有限公司, 广州 510632) \\ (3: 中国科学院南京地理与湖泊研究所, 南京 210008)
}

\begin{abstract}
摘 要: 热带浅水湖泊后生浮游动物群落以轮虫和小型枝角类为主, 往往缺乏大型浮游动物, 其影响因子还存在争议, 因此 通过减少鱼类, 提高大型后生浮游动物密度, 从而增强对浮游植物的下行控制、修复热带富营养化湖泊的方法受到质疑. 暨大 南湖位于广州暨南大学校园内, 是热带富营养化浅水湖泊, 于 2014 年 1-2 月实施以鱼类去除和水生植被重建为主的生态系 统修复工程, 以期改善水质. 通过对南湖修复前后水质、后生浮游动物以及修复后鱼类群落的调查分析, 研究了后生浮游动物 群落对修复的响应. 结果表明: 与修复前相比, 总氮、总磷和叶绿素 a 浓度显著降低, 透明度显著上升. 枝角类丰度和生物量显 著增加, 修复后初期出现较高密度的大型枝角类蚤状溞 (Daphnia pulex), 桡足类丰度和生物量没有显著变化, 轮虫丰度下降 而生物量上升; 后生浮游动物体长和生物多样性显著增加.随着鱼类密度的增加, 蚤状溞丰度快速降低, 后期鱼类减少后蚤状 溞丰度又有所升高. 因此, 本研究显示鱼类是控制热带浅水湖泊大型浮游动物种群的主要因素, 由于控制鱼类密度以维持较 高大型浮游动物密度较为困难,因此单纯生物操纵在热带浅水湖泊修复中难以取得显著效果.
\end{abstract}

关键词: 热带浅水湖泊; 后生浮游动物;生态修复;鱼类;群落结构

\section{Response of metazoan zooplankton communities to ecological restoration in a tropical shallow lake}

\author{
ZENG Haiyi ${ }^{1}$, ZHONG Ping ${ }^{1}$, ZHAO Xuefeng ${ }^{2}$, LI Chao ${ }^{2}$, HE Xiaolin ${ }^{2} \&$ LIU Zhengwen ${ }^{1,3 * *}$ \\ (1: Department of Ecology and Institute of Hydrobiology, Jinan University, Guangzhou 510632, P.R.China) \\ (2: Belsun Environmental Protection, Guangzhou 510632, P.R. China) \\ (3: Nanjing Institute of Geography and Limnology, Chinese Academy of Sciences, Nanjing 210008, P.R.China)
}

\begin{abstract}
The metazoan zooplankton communities were dominated by small cladocerans and rotifers, often lack of large-sized zooplankton in tropical lakes and the controlling factors are not clear. Thus it has been questioned if eutrophic lakes can be restored by reducing fish, increasing large-sized metazoan zooplankton, and the top-down control on phytoplankton in the tropics. South Lake from Jinan University, Guangzhou, is a tropic eutrophic shallow lake. The lake was restored via fish removal and plantation of submerged macrophytes in order to improve water quality. We monitored the water quality, metazoan zooplankton communities and fish before and after restoration in order to study the response of metazoan zooplankton communities to the restoration. The results showed that total nitrogen, total phosphorus and chlorophyll-a concentrations decreased significantly and the transparency increased. The abundance and biomass of cladoceran increased, and high numbers of large-sized cladoceran Daphnia pulex were observed shortly after the restoration. The abundance and biomass of copepods had no significant changes. The density of rotifers decreased, but biomass increased. The sizes of metazoan zooplankton and biodiversity increased. The abundance of Daphnia pulex decreased quickly when fish biomass increased, and increased again when fish biomass was reduced later. Therefore, this study suggests that fish is a key factor controlling the population of large-sized zooplankton in tropical shallow lakes. As controlling fish to maintain high densities of large-sized zooplankton is difficult, it is unlikely to achieve a significant improvement in the restoration of tropical lakes using only biomanipulation. Keywords: Tropical shallow lake; metazoan zooplankton; ecological restoration ; fish ; community structure
\end{abstract}

* 国家重点基础研究发展计划 “973” 项目 (2012CB956100) 资助.2014-12-18 收稿; 2015-04-27 收修改稿. 曾海逸 (1990 ), 男, 硕士研究生;E-mail :zenghaiyi2000@ sina.com.

** 通信作者;E-mail :zliu@ niglas.ac.cn. 
由氮、磷等营养盐的过度输人而引起的水体富营养化已成为世界上最受关注的水环境问题之一 ${ }^{[1]}$.近年 来随着社会经济发展和人口膨胀, 湖泊富营养化日趋严重. 湖泊富营养化往往导致蓝藻过度繁殖形成水 华 ${ }^{[2-4]}$, 使得水体透明度下降, 生物多样性降低, 生态系统的结构和功能严重退化 ${ }^{[5]}$. 富营养化水体治理首先 要削减营养盐负荷, 包括点源和面源 ${ }^{[6-7]}$, 但是沉积物再悬浮和营养盐释放可形成较高的内源营养盐负荷, 水质往往达不到预期的效果 ${ }^{[8-10]}$. 温带湖泊的研究表明, 通过生物操纵大幅减少食浮游生物鱼类密度, 可以 提高大型浮游动物密度, 有效控制浮游植物的生长, 改善水质 ${ }^{[11-12]}$. 因此, 生物操纵方法已成为温带富营养化 湖泊修复的主要手段之一 ${ }^{[13]}$.

温带湖泊浮游动物, 尤其是大型枝角类如溞属 (Daphnia $)^{[14-15]}$, 是浮游植物的主要牧食者, 从而成为控 制 “水华” 发生的主要因素之一 ${ }^{[16]}$. 而食浮游生物鱼类的捕食往往导致浮游动物群落结构小型化 ${ }^{[17]}$, 在鱼类 密度较高时, 大型浮游动物难以生存, 浮游动物对浮游植物的牧食压力较小. 当通过放养肉食性鱼类或直接 捕捞等方法降低食浮游生物鱼类密度时, 浮游动物群落结构会迅速响应, 大型浮游动物密度显著上升, 从而 使浮游动物对浮游植物的下行控制能力提高, 浮游植物密度降低, 从而促进沉水植物的恢复和水质改善. 通 过上述方法 (即生物操纵) 修复富营养化湖泊在温带地区得到了较广泛的应用.

然而, 生物操纵在较温暖的热带、亚热带水体的效果遭到质疑 ${ }^{[5,13]}$. 与温带相比, 在相似营养水平下热带、 亚热带湖泊具有更高的生产力 ${ }^{[18]}$, 浮游动物密度较低, 而且个体较小 ${ }^{[19]}$. 有研究表明, 虽然通过鱼类调控和沉 水植物修复, 热带浅水湖泊水质同样得到显著改善, 但浮游动物密度仍较低, 尤其缺乏大型浮游动物, 如溞属 种类 ${ }^{[20]}$. 有的学者认为, 热带、亚热带湖泊鱼类密度更高, 尤其是个体较小的鱼类密度周年维持较高的水平, 难 以通过生物调控降到较低的水平, 因此浮游动物群落对生态修复的响应不显著 ${ }^{[13]}$. 也有学者认为, 热带水体较 高的水温是造成大型浮游动物贫乏的重要因子 ${ }^{[21]}$. 因此, 目前对热带、亚热带湖泊浮游动物群落的控制因子还 存在争议, 浮游动物在控制热带、亚热带湖泊浮游植物生长和湖泊修复中的作用仍不清楚 ${ }^{[13]}$.

位于广州市暨南大学内的南湖 $\left(23^{\circ} 07^{\prime} \mathrm{N}, 113^{\circ} 20^{\prime} \mathrm{E}\right)$ 是个人工湖, 建于 1958 年, 面积为 $4000 \mathrm{~km}^{2}$, 平均 水深约 $1.0 \mathrm{~m}$. 由于周围生活污水和地表径流的污染, 水体富营养化严重. 为彻底整治南湖, 于 2013 年初进行 了截污,2014 年 1 月实施了以鱼类去除和沉水植物群落重建为主的生态修复工程: 首先去除浮游生物食性 鱼类和底栖杂食性鱼类,包括罗非鱼 (Oreochomis sp.)、鲤(Cyprinus carpio) 和鲫 (Carassius auratus) 等; 种植了 沉水植物, 包括苦草 (Vallisneria natans) 、轮叶黑藻 (Hydrilla verticillata) 及穗花狐尾藻 (Myriophyllum spicatum) 等,其中以苦草为优势种,覆盖率在 2 月份约为 $25 \%, 9$ 月份增至约 $85 \%$.

本文通过比较南湖修复前后后生浮游动物群落结构的变化, 分析了浅水湖泊后生浮游动物对湖泊生态 修复的响应, 并探讨了浮游动物在热带湖泊中的作用, 结果有助于揭示热带湖泊浮游动物结构的控制因子.

\section{1 材料与方法}

\section{1 采样时间和地点}

在暨南大学南湖的北部、中部和南部各设置 1 个采样点, 分别于 2013 年 3-9 月 (修复前) 和 2014 年 3-9 月 (修复后) 进行常规水样和后生浮游动物的采集,鱼类样品采集从 2014 年 3 月底开始.

\section{2 水样的采集及分析}

常规水质样品的采集与分析按照《湖泊生态调查观测与分析》 ${ }^{[22]}$ 进行: 采用 $5 \mathrm{~L}$ 有机玻璃采水器采集, 将水样低温保存, 带回实验室进行理化指标测定, 包括总氮 (TN)、总磷 (TP) 和叶绿素 a (Chl.a) 浓度等, 透明 度 $(\mathrm{SD})$ 现场采用塞氏盘测定.

\section{3 后生浮游动物及鱼类的采集}

后生浮游动物样品采集采用 $5 \mathrm{~L}$ 采水器于水面下从上至下均匀采集 $20 \mathrm{~L}$, 混匀后取水 $1 \mathrm{~L}$, 带回实验室, 静置、沉淀、浓缩, 用于轮虫定量分析; 其余水样用 $25^{\#}$ 网过滤、浓缩, 用于浮游甲壳类定量分析. 所有后生浮游 动物样品均于采样现场加人 $5 \%$ 福尔马林固定后带回实验室处理.

轮虫按照《中国淡水轮虫志》 ${ }^{[23]}$ 鉴定; 浮游甲壳类按照《中国动物志》 ${ }^{[24-25]}$ 鉴定. 根据浮游动物体积的近似 计算公式 ${ }^{[26-27]}$, 测定轮虫、枝角类和桡足类的体长、体宽或直径等以计算每个个体体积, 并认为浮游动物的密 度与水 $(1 \mathrm{~g} / \mathrm{ml})$ 相同, 从而得出每个个体的体重, 最后计算浮游动物的生物量. 
鱼类样品用刺网 (网孔大小分别为 30 和 $60 \mathrm{~mm}$; 长 $20 \mathrm{~m}$ 、高 $1.2 \mathrm{~m}$ ) 进行采集, 1 周 2 次. 在各采样点采集 鱼类时, 于傍晚放网, 保持刺网在水中停留 $12 \mathrm{~h}$, 次日上午起网后摘取捕获的鱼类, 进行分类、计数, 并测量 体长和体重.种类鉴定参照《广东省淡水鱼类志》 ${ }^{[28]}$.

\section{4 数据分析及处理}

后生浮游动物的生物多样性采用 Shannon-Wiener 多样性指数 $\left(H^{\prime}\right)$ 进行评价:

$$
H^{\prime}=-\sum_{i=1}^{s} \frac{n_{i}}{N} \cdot \log _{2} \frac{n_{i}}{N}
$$

式中, $n_{i}$ 为第 $i$ 种后生浮游动物的密度, $N$ 为后生浮游动物总密度 (ind. $/ \mathrm{L}$ ).

优势种采用优势度 $\left(D_{i}\right)$ 进行评价:

$$
D_{i}=\frac{n_{i}}{N} \times 100 \%
$$

式中, $n_{i}$ 为第 $i$ 种的数量, $N$ 为该群落所有种的数量.

各理化因子以及后生浮游动物丰度之间的差异采用 SPSS 22.0 软件进行方差分析.

\section{2 结果}

\section{1 修复前后水体理化特征比较}

修复后水体 $\mathrm{TN}$ 平均浓度为 $0.80 \mathrm{mg} / \mathrm{L}$, 显著低于修复前的 $2.81 \mathrm{mg} / \mathrm{L}(P<0.05)$; 修复后 $\mathrm{TP}$ 平均浓度为 $0.05 \mathrm{mg} / \mathrm{L}$, 显著低于修复前的 $0.12 \mathrm{mg} / \mathrm{L}(P<0.05)$; 修复后 $\mathrm{Chl} . \mathrm{a}$ 平均浓度为 $17.88 \mu \mathrm{g} / \mathrm{L}$, 显著低于修复前的 125.62 $\mu \mathrm{g} / \mathrm{L}(P<0.05)$; 而修复后平均 $\mathrm{SD}$ 为 $93.74 \mathrm{~cm}$,显著高于修复前的 $36.6 \mathrm{~cm}(P<0.05)$ (图 1).
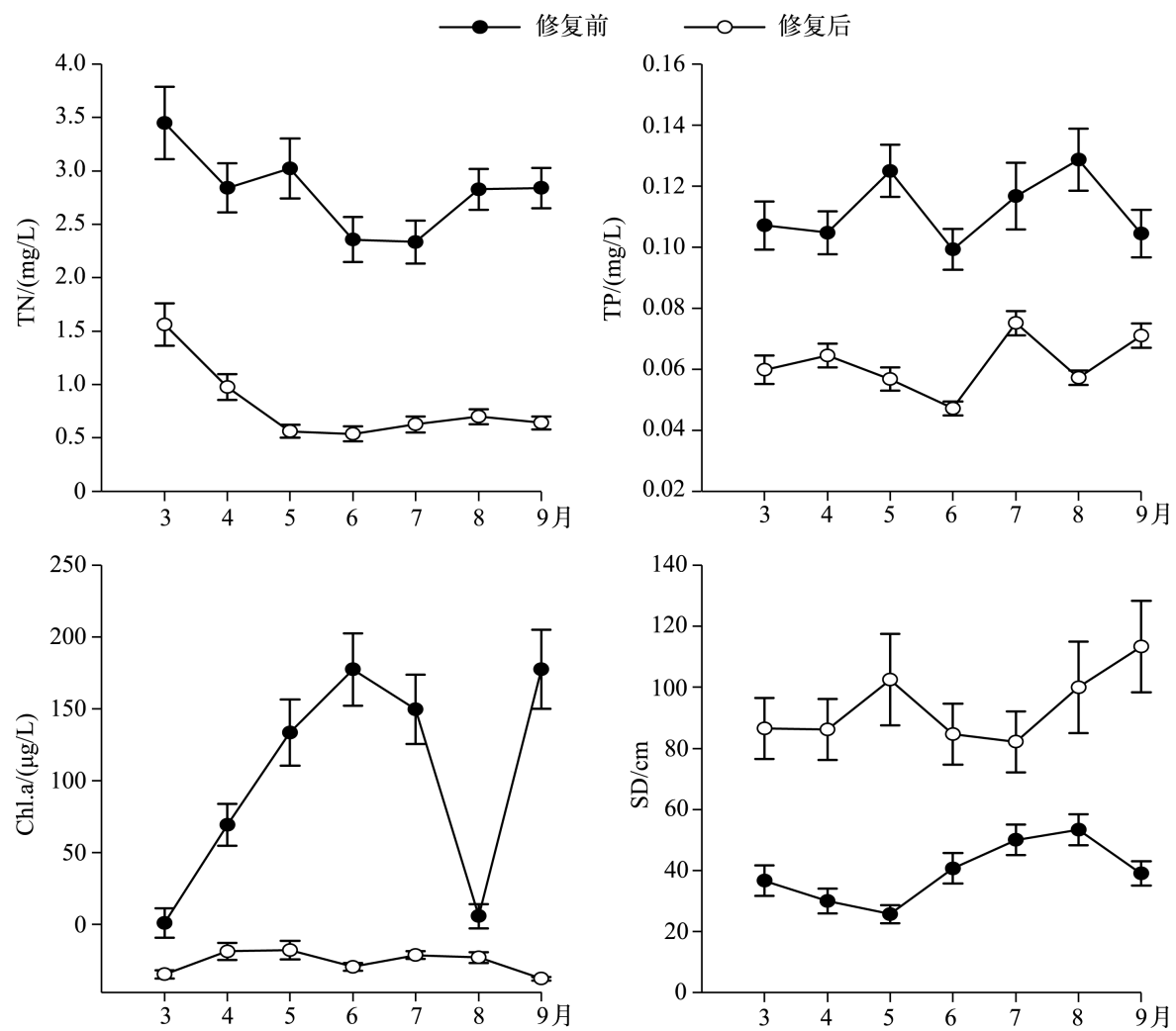

图 1 暨南大学南湖修复前后水体总氮、总磷、叶绿素 a 浓度和透明度

Fig.1 Total nitrogen, total phosphorus, chlorophyll-a concentrations and transparency before and after the restoration in the South Lake of Jinan University 


\section{2 后生浮游动物}

2.2.1 后生浮游动物种类组成 共检出轮虫 20 种, 其中修复前 8 种, 优势种属包括臂尾轮虫 (Brachiorms sp.) 和热带龟甲轮虫 (Keratella tropica); 修复后水体中检出 17 种, 优势属为多肢轮虫 (Polyarthra sp.). 裂足臂尾 轮虫 (Brachionus diversicornis)、镰状臂尾轮虫(B.falcatus) 和蹄型腔轮虫 (Lecane ungulata) 仅在修复前出现; 检出浮游甲壳动物 9 种, 其中修复前 3 种, 优势种为模糊秀体溞 (Diaphnnosoma dubium) 和台湾温剑水蚤 (Thermocyclops taihokuensis); 修复后检出 9 种, 优势种包括蚤状溞 (Daphnia pulex) 和台湾温剑水蚤.蚤状溞、 奥氏秀体溞 (Diaphanosoma orghidani)、角突网纹溞(Ceriodaphnia cornuta)、长额象鼻溞( Bosmina longirostris) 和奇异尖额溞 (Alona eximia) 仅在修复后出现,未发现哲水蚤.

2.2 .2 后生浮游动物丰度、生物量及生物多样性 修复后枝角类丰度及生物量显著高于修复前 $(P<0.05)$; 轮 虫丰度显著低于修复前 $(P<0.05), 4$ 月份开始生物量显著高于修复前; 修复前后桡足类无显著变化 ( 图 2 、 $3)$. 修复后 3 月初开始, 虫状溞大量出现, 直到 5 月初逐渐消失, 9 月初蚤状溞再度出现,但丰度较低, 保持在 15 30 ind./L 左右 ( 图 2). 对后生浮游动物多样性分析可知, 修复后浮游动物 Shannon-Wiener 多样性指数显 著高于修复前 $(P<0.05)$ ( 图 4).

2.2.3 后生浮游动物体长 修复前浮游动物体长分布在 $0.1 \sim 1.1 \mathrm{~mm}$, 平均体长为 $0.69 \mathrm{~mm}$; 修复后体长则分 布在 $0.12 \sim 2.0 \mathrm{~mm}$, 平均体长为 $0.71 \mathrm{~mm}$. 其中修复前浮游动物体长主要分布在 $0.1 \sim 0.8 \mathrm{~mm}$, 其中体 长 $<0.6 \mathrm{~mm}$ 的个体占 $76 \%$. 而修复后体长主要分布在 $0.2 \sim 1.2 \mathrm{~mm}$, 其中体长 $>0.6 \mathrm{~mm}$ 的个体约占 $56 \%$ ( 图 5).
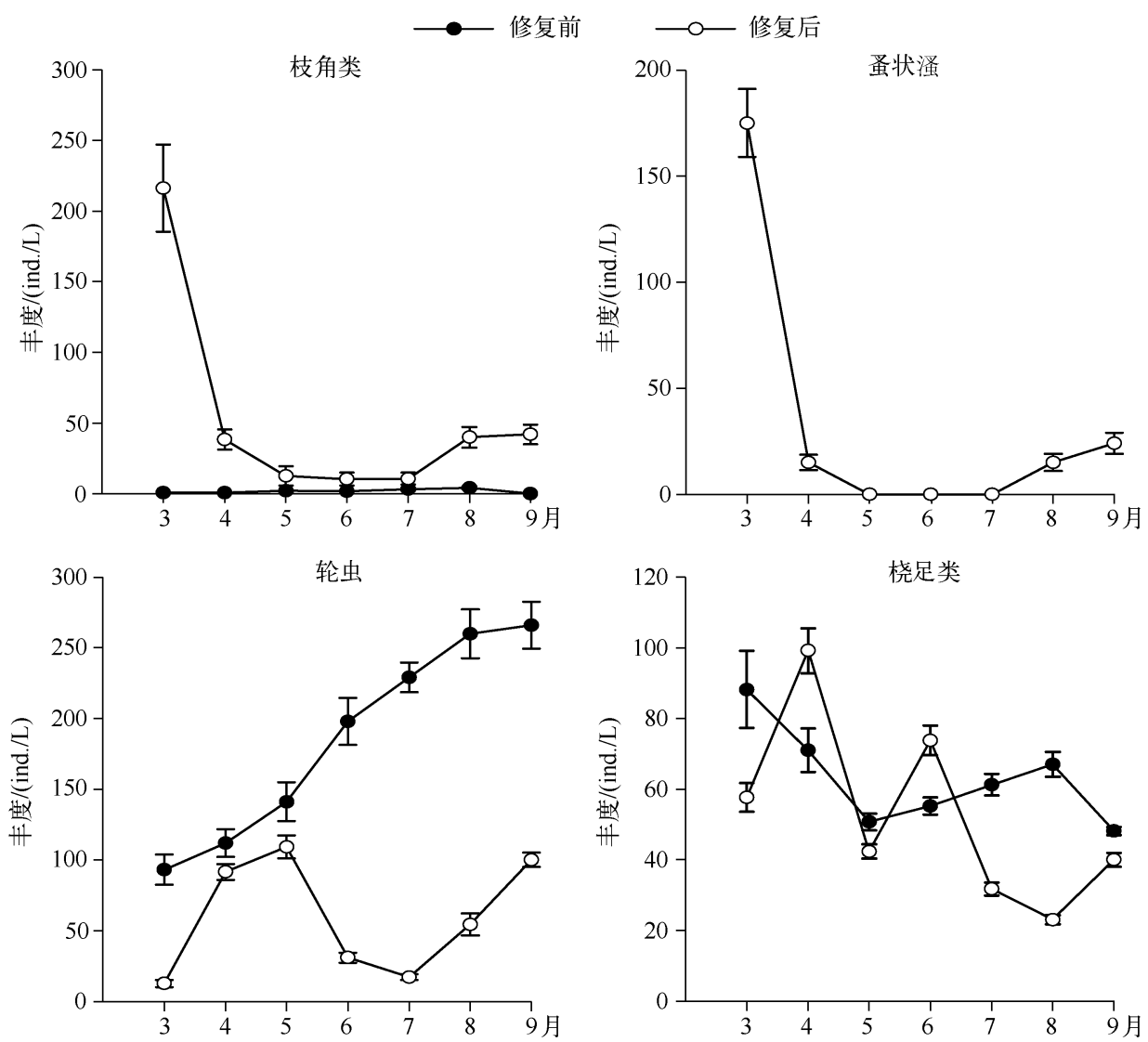

图 2 暨南大学南湖修复前后后生浮游动物丰度比较

Fig. 2 The metazoan zooplankton abundance before and after the restoration in the South Lake of Jinan University 

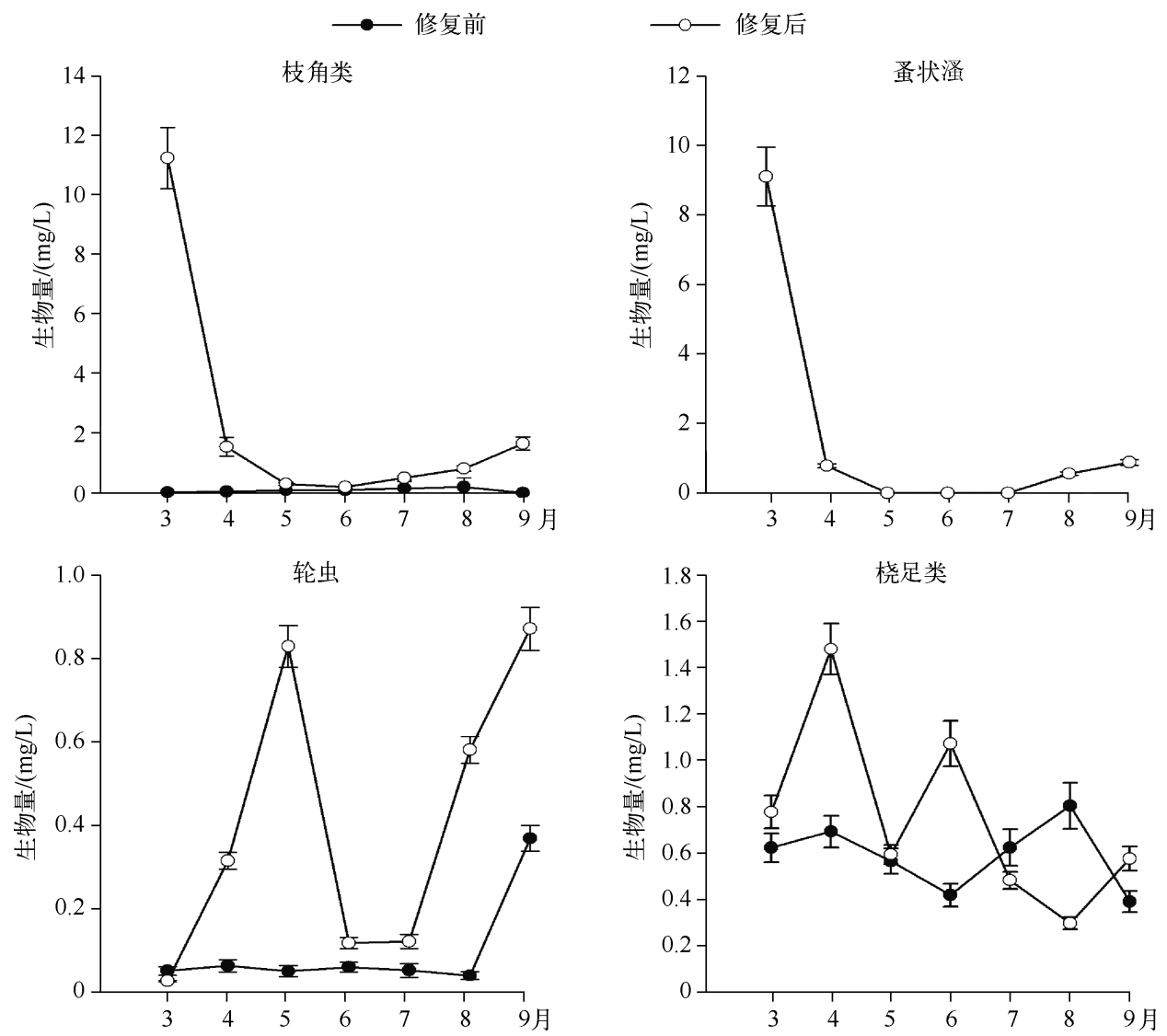

图 3 暨南大学南湖修复前后后生浮游动物生物量比较

Fig. 3 The metazoan zooplankton biomass before and after the restoration in the South Lake of Jinan University

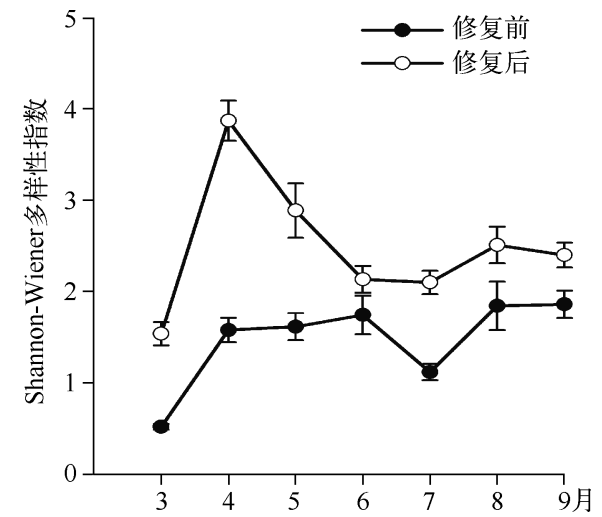

图 4 暨南大学南湖修复前后后生浮游 动物多样性指数比较

Fig.4 The diversity index of metazoan zooplankton before and after the restoration in the South Lake of Jinan University

\section{3 鱼类}

修复后共捕获鱼类 16 种, 主要有罗非鱼、鲤、鲶 (Silurus asotus)、鲫、泥鳅(Misgurnus anguillicaudatus)、黄鳝 (Monopterus albus) 等, 以罗非鱼和鲫为主, 其中罗非鱼体长 主要分布在 $5 \sim 18 \mathrm{~cm}$ 之间, 鲫体长主要分布在 $4 \sim 8 \mathrm{~cm}$ 之间.

修复后每次捕获鱼类的数量显著增加, 从 3 月的 18 ind./(网· $12 \mathrm{~h}$ ) 增加至 8 月的 168 ind./(网・12 h), 呈总 体上升的趋势, 而在此期间渔获量 (重量) 变化不如数量变 化显著, 8 月底进行大规模捕鱼, 9 月渔获量为 0 (图 6).

\section{3 讨论与结论}

修复后的暨大南湖水生植被覆盖率增至 $85 \%$ 左右, 水 体营养盐和 Chl.a 浓度显著降低, 透明度显著增加, 水质得 到显著改善. 后生浮游动物种属数、多样性和个体大小都显 著提高. 其中枝角类丰度和生物量显著上升, 轮虫丰度下降 
而生物量上升, 桡足类无明显变化. 修复前优势种 包括臂尾轮虫、热带龟甲轮虫和裸腹溞等. 而修复 后的优势种为多肢轮虫、蚤状溞、台湾温剑水虫和 长额象鼻溞等. 臂尾轮虫、裸腹溞和长额象鼻溞等 种类的变化与陈光荣等 ${ }^{[29]}$ 报道的惠州西湖的结 果相似.而南湖在修复初期虽然出现了大量虫状 溞, 但很快数量下降, 甚至消失, 这可能与热带湖 泊中鱼类恢复较快, 而沉水植物对浮游动物的庇 护作用并不明显有关 ${ }^{[30-31]}$.

与温带湖泊相比,热带湖泊中鱼类个体较小、 食浮游动物鱼类比例较高 ${ }^{[32]}$. 暨南大学南湖修复 后出现的鱼类以小型罗非鱼和鲫鱼为主, 这些鱼 类繁殖速度快, 而且一年繁殖多次, 幼鱼数量全年 较多, 浮游动物是其主要食物, 尤其喜食大型浮游 动物 ${ }^{[32]}$. 修复后浮游动物体长有所增加, 说明浮 游动物所受到的捕食压力小于修复前 ${ }^{[33]}$. 修复后 暨大南湖轮虫丰度下降, 而生物量上升, 主要是因 为轮虫个体增大, 而个体增大无疑与鱼类捕食压 力下降有关 ${ }^{[34]}$. 桡足类逃避鱼类捕食能力较强, 因 此其密度的变化往往与鱼类密度关系不大 ${ }^{[35]}$, 这 可能是导致南湖修复前后桡足类差别不显著的主 要原因.大型枝角类虫状溞只在初期丰度较高, 后 期密度很低, 这主要是鱼类群落快速恢复造成的. 许多研究表明, 热带鱼类种类比温带丰富 ${ }^{[36]}$, 大 型肉食性鱼类较少, 杂食性鱼类往往占优势 ${ }^{[37]}$. 同 时, 由于热带湖泊水温常年较高, 许多鱼类都能够 全年繁殖 ${ }^{[38-39]}$, 暨南大学南湖修复后渔获量 (重 量) 增加小于数量增加的趋势 (图 6), 主要是个体 较小的鱼类比例上升, 而小个体鱼类对浮游动物 的捕食压力较高 ${ }^{[40]}$. Jeppesen 等 ${ }^{[33]}$ 认为热带湖泊 中小个体杂食性鱼类占据优势且常聚集在沉水植 物植被区, 这些鱼类大量摄食浮游动物, 尤其喜食 大型浮游动物 ${ }^{[41]}$, 水生植物群落起不到保护大型

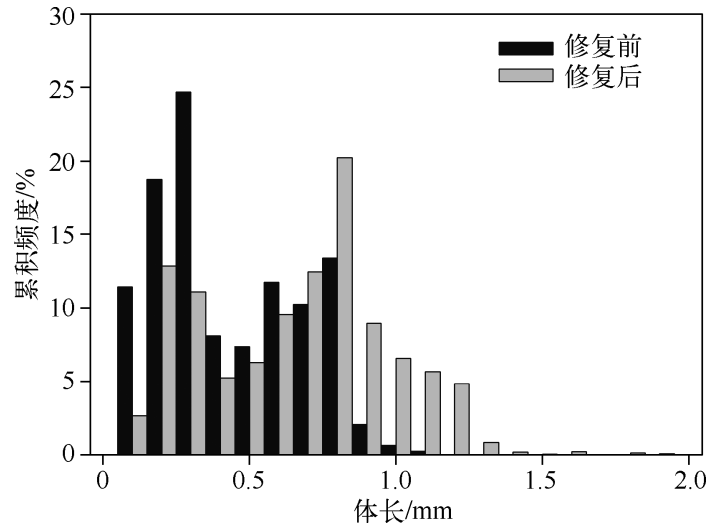

图 5 暨南大学南湖修复前后后生浮游 动物月均体长的累积频度

Fig. 5 The cumulative frequency of metazoan zooplankton body size before and after the restoration in the South Lake of Jinan University

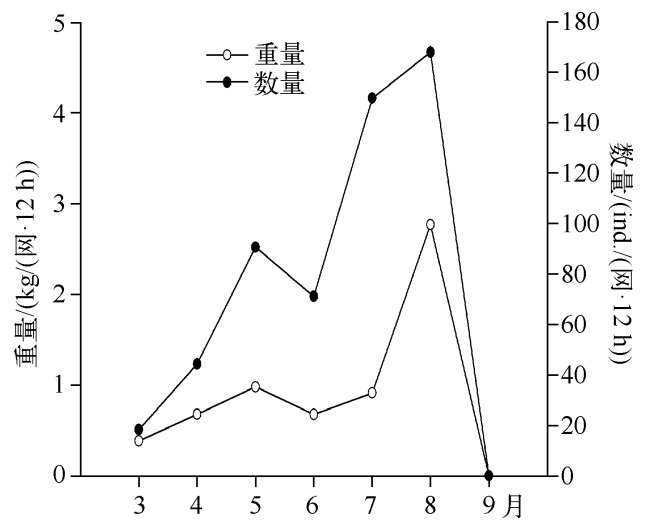

图 6 暨南大学南湖修复后渔获量 (数量及重量)

Fig. 6 The fish catches (numbers and weights) after the restoration in the South Lake of Jinan University

浮游动物庇护所的功能, 因此对浮游动物的捕食压力常年较高. 本研究中于 8 月底调整了捕鱼方法, 对鱼类 进行大量捕捞后, 蚤状溞再度出现 (图 2), 这说明在热带浅水湖泊中, 鱼类是控制大型浮游动物种群变化的

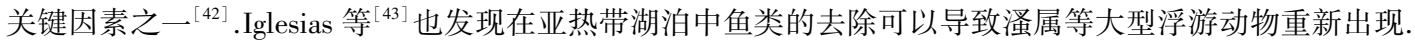

由此可见, 鱼类去除和沉水植被恢复能有效改善热带湖泊水质, 提高后生浮游动物多样性, 恢复大型浮 游动物种群,包括虫状溞.但鱼类种群的快速恢复会使大型浮游动物种群迅速减少,甚至消失. 因此,在热带 湖泊中控制鱼类种群规模以维持较高大型浮游动物种群丰度较为困难, 控制浮游植物的下行效应较弱, 经 典的生物操纵在热带浅水湖泊修复中难以取得显著效果.

\section{4 参考文献}

[ 1 ] Schindler DW. Recent advances in the understanding and management of eutrophication. Limnology and Oceanography, 2006, 51 (1) : 356-363. 
[ 2 ] Jeppesen E, Jensen JP, Kristensen P et al. Fish manipulation as a lake restoration tool in shallow, eutrophic, temperate lakes 2: threshold levels, long-term stability and conclusions. Hydrobiologia, 1990, 200: 219-227.

[ 3 ] Jeppesen E, Jensen JP, Søndergaard M et al. Top-down control in freshwater lakes: the role of nutrient st-ate, submerged macrophyte sand water depth. Hydrobiologia, 1997, 342: 151-164.

[ 4 ] Willame RT, Jurczak JF, Iffly T et al. Distribution of hepatotoxic cyanobacterial blooms in Belgium and Luxembourg. Hydrobiologia , 2005, 551: 99-117.

[ 5 ] 刘正文. 湖泊生态系统恢复与水质改善. 中国水利, 2006, (17) : 30-33.

[ 6 ] Jeppesen E, Jensen JP, Søndergaard M et al. Response of fish and plankton to nutrient loading reduction in eight shallow Danish lakes with special emphasis on seasonal dynamics. Freshwater Biology, 2005, 50: 1616-1627.

[ 7 ] Jeppesen E, Sondergaard M, Meerhoff M et al. Shallow lake restoration by nutrient loading reduction-some recent findings and challenges ahead. Hydrobiologia, 2007, 584: 239-252.

[ 8 ] Lauridsen TL, Jensen JP, Jeppesen E et al. Response of submerged macrophytes in Danish lakes to nutrient loading reductions and biomanipulation. Hydrobiologia, 2003, 506: 641-649.

[ 9 ] Carpenter SR. Eutrophication of aquatic ecosystem: bistability and soil phosphorus. PNAS, 2005, 102: 1002-1005.

[10] Søndergaard M, Jeppesen E, Lauridsen TL et al. Lake restoration: successes, failures and long-term effects. Journal of Applied Ecology, 2007, 44: 1095-1105.

[11] Moss B, Madgwick J, Phillips G. A guide to the restoration of nutrient-enriched shallow lakes. UK: Broad Authority \& Environment Agency, 1996: 1-179.

[12] Lammens EHHR. The central role of fish in lake rest-oration and management. Hydrobiologia, 1999, 395/396: 191-198.

[13] Jeppesen E, Søndergaard M, Lauridsen TL et al. Biomanipulation as a restoration tool to combat eutrophication: recent advances and future challenges. In: Woodward G ed. Advances in ecological research. New York: Elsevier Academic Press Inc, 2012, 47: 411-488.

[14] Moss B, Stansfield J, Irvine K et al. Progressive restoration of a shallow lake: A 12-year experiment in isolation, sediment removal and biomanipulation. Journal of Applied Ecology, 1996, 33: 71-86.

[15] Meijer ML, De Boois I, Scheffer M et al. Biomanipulation in shallow lakes in the Netherlands: an evaluation of 18 case studies. Hydrobiologia, 2003, 408/409: 13-30.

[16] Ghadouani A, Pinel-Alloul B, Prepas EE. Effects of experimentally induced cyanobacterial blooms on crustacean zooplankton communities. Freshwater Biology, 2003, 48: 363-381.

[17] Carpenter SR, Kitchell JF, Hodgson JR. Cascading trophic interactions and lake productivity. Bioscience, 1985, 35( 10) : 634-639.

[18] Beklioglu M, Meerfhoff M, Søndergaard M et al. Eutrophication and restoration of shallow lakes from a cold temperate to a warm Mediterranean and a (sub) tropical climate. Eutrophication: causes, consequences and control. Dordrecht: Springer Netherlands, 2011: 91-108.

[19] Havens K, Elia A, Taticchi M et al. Zooplankton-phytoplankton relationships in shallow subtropical versus temperate lakes Apopka (Florida, USA) and Trasimeno(Umbria, Italy). Hydrobiologia, 2009, 628: 165-175.

[20] 陈光荣, 刘正文, 钟 萍等. 热带城市湖泊生态恢复中水生植被、浮游动物和鱼类的关系研究. 生态环境, 2007, 16: $1-7$.

[21] Sarma SSS, Nandini S, Gulati RD. Life history strategies of cladocerans: comparisons of tropical and temperate taxa. Hydrobiologia, 2005, 542(1): 315-333.

[22] 黄祥飞. 湖泊生态调查观测与分析. 北京: 中国标准出版社, 1999.

[23] 王家楫. 中国淡水轮虫志. 北京: 科学出版社, 1961 .

[24] 蒋譍治, 堵南山. 中国动物志 - 淡水枝角类. 北京: 科学出版社, 1979.

[25] 沈嘉瑞. 中国动物志 - 淡水桡足类. 北京: 科学出版社, 1979.

[26] 章宗涉, 黄祥飞. 淡水浮游生物研究. 北京: 科学出版社, 1991.

[27］陈雪梅. 淡水桡足类生物量的测算. 水生生物学集刊, 1981, 7(3) : 397-408.

[28] 潘畑华, 钟 麟, 郑慈英等. 广东淡水鱼类志. 广东: 广东科技出版社, 1991.

[29] 陈光荣, 钟 萍, 张修峰等. 惠州西湖浮游动物及其与水质的关系. 湖泊科学, 2008, 20(3): 351-356. DOI 10. 18307/2008. 0314. 
[30] Meerhoff M, Clemente JM, Teixeira-de Mello F et al. Can warm climate-related structure of littoral predator assemblies weaken the clear water state in shallow lakes? Global Change Biology, 2007, 13: 1888-1897.

[31] Meerhoff M, Iglesias C, Teixeira de Mello F et al. Effects of habitat complexity on community structure and predator avoidance behaviour of littoral zooplankton in temperate versus subtropical shallow lakes. Freshwater Biology, 2007, 52: 1009-1021.

[32] Lazzaro X. Do the trophic cascade hypothesis and classical biomanipulation approaches apply to tropical lakes and reservoirs? Verhandlungen -Internationale Vereinigung fiur theoretische und angewandte Limnologie, 1997, 26: 719-730.

[33] Jeppesen E, Sondergaard M, Mazzeo N et al. Lake restoration and biomannipulation in temperate lakes: relevance for subtropical and tropical lakes. In: Reddy V ed. Tropical eutrophic lakes: Their restoration and management( in press), 2006.

[34] Stenson JAE. Fish impact on rotifer community structure. Hydrobiologia, 1982, 87(1) : 57-64.

[35] Lazzaro X. A review of planktivorous fishes: their evolution, feeding behaviours, selectivities, and impacts. Hydrobiologia, 1987, 146(2) : 97-167.

[36] Lévêque C. Biodiversity dynamics and conservation: the freshwater fish of tropical Africa. Transactions of the American Fisheries Society, 1997, (1): 182-183.

[37] Teixeira-de Mello F, Meerhoff M, Pekcan-Hekim Z et al. Substantial differences in littoral fish community structure and dynamics in subtropical and temperate shallow lakes. Freshwater Biology, 2009, 54: 1202-1215.

[38] Fernando CH. Zooplankton, fish and fisheries in tropical freshwaters. Hydrobiologia, 1994, 272: 105-123.

[39] Paugy D, Le'vêque C. La Reproduction. In: Paugy D, Le'vêque C eds. Les poisons des eaux continentales africaines: Diversite', e'cologie, utilisation par l'homme. Paris: IRD Editions, 1999: 129-152.

[40] Jeppesen E, Meerhoff M, Holmgren K et al. Impacts of climate warming on lake fish community structure and potential effects on ecosystem function. Hydrobiologia, 2010, 646(1) : 73-90.

[41] Kalff J. Limnology-Inland water ecosystems. New Jersey: Upper Saddle River, Prentice-Hall Inc, 2002.

[42] Fernando CH. Zooplankton and tropical freshwater fisheries. Leiden: Backhuys Publishers, 2002: 255-280.

[43] Iglesias C, Mazzeo N, Meerhoff M et al. High predation is the key factor for dominance of small-bodied zooplankton in warm lakes-evidence from lakes, fish enclosures and surface sediment. Hydrobiologia, 2011, 667: 133-147. 